Hilko Weerda

\title{
Surgery of the Auricle
}

Tumors, Trauma, Defects, Abnormalities

\section{Thieme, Stuttgart 2007}

320 pp.; EUR 179.95

ISBN 978-3-13-139411-8

This book highlights all surgical procedures associated with the external ear. It is divided into 6 main chapters: (1) basic principles; (2) tumors of the external ear; (3) trauma and noninflammatory processes; (4) classification and surgery of auricular defects; (5) abnormalities, and (6) diagnostics and auxiliary therapy.

The strength of this book is how the editor addresses both practical issues for beginner clinicians and advanced issues for very experienced clinicians, in the broad field from small traumas to total ear reconstructions. Before-and-after photos illustrate the clinical opportunities using the appropriate surgical approach.

Helpful hints are given for reconstruction of small defects preserving the shape and dimension of the auricle. These techniques are applied to every part of the ear.

Using the embryological development, the most common auricular malformations are explained. Based on this knowledge, the surgical correction is shown in detail including a variety of techniques for the correction of the protruding ear as well as possibilities for the reconstruction of secondary deformations due to otoplasty. This section ends with the possibilities of complete ear reconstruction using autogenous rib cartilage. The technique is excellently described in detail, and the highly satisfying results elucidate the importance of reconstruction of the auricle already in childhood.

This book is a compilation of the vast and outstanding experience of the editor specializing in treating defects and disorders of the auricle. It succeeds beautifully in capturing scientific and technical facts of ear reconstruction. The more than 1,300 detailed photographs and illustrations provide a perfect understanding of anatomy, morphology and the surgical procedures. Concise descriptions and illustrated step-by-step instructions enable surgeons to successfully operate in this field.

R. Staudenmaier, Munich

\section{KARGER

(C) 2008 S. Karger AG, Basel 